\title{
Classical Theory of Detonation
}

\author{
W.C. Davis and C. Fauquignon*
}

Energetic Dynamics Los Alamos, 693 46th Street, Los Alamos, NM 87544, U.S.A.

* French-German Research Institute, 5 rue du Général Cassagnou, 68301 Saint-Louis cedex, France

\begin{abstract}
In a first part is presented the model of the Ideal Detonation. Emphasis is placed on the physical assumptions made in the setting and resolution of the continuum mechanics equations to be used. The basic elements of computations of ideal detonation parameters will be described. The experiments performed to check the predictions, and their results, will be reported. Some discussion of explosives in which chemical equilibrium is not reached, due to slow diffusive mixing of the reactants or to conditions where some of the reactions are very slow, will also be given. These explosives are often called non-ideal explosives. Obviously, when the reaction zone is not very thin relative to system dimensions, or when it is not very short relative to system times, the Ideal Detonation model, restricted to plane, steady flow, is inadequate. The third part of the paper is concerned with curved detonation fronts, and with time dependent processes. The steady detonation of small-diameter cylindrical cartridges will be discussed as one example, and the initiation of detonation in the shock-to-detonation transition as another. Detonation theory is not a closed subject, and much effort is currently being spent to extend classical theory. Some of the newer ideas will be introduced in a concluding part.
\end{abstract}

\section{THERMO-HYDRODYNAMIC DESCRIPTION OF DETONATION (C. Fauquignon)}

\subsection{Introduction}

An explosive is defined as a substance which can deliver very quickly a large amount of energy and produce a large amount of gas. The energy has generally a chemical origin and the reactions progress in the surrounding material by drawing a part of the energy previously produced. 
According to the processes of the energy transfer in the non reacted material, two regimes are defined, deflagration and detonation, which are differentiated from the usual combustion by their velocities of propagation (some $\mathrm{mm} / \mathrm{s}$ for the usual combustion, several hundred $\mathrm{m} / \mathrm{s}$ for the deflagration and several thousand $\mathrm{m} / \mathrm{s}$ for the detonation). It follows that the flow of the high pressure reaction products is the acting agent of the energy transfer and of the self sustaining of the combustion. As a consequence, the description of the process calls in the fluids mechanics associated with the thermo-chemistry for the setting of the energy balance.

In this presentation, we will only consider the case of the detonation in which, contrary to the deflagration, the energy transfer in the non reacted material is supersonic. The detonation is characterised by the existence of a shock wave in the front of which the reactions start.

The model of the Ideal thermo-hydrodynamic Detonation which will be presented does not take into account the physico-chemical processes in the shock front or in its neighbourhood and which may require revision of the assumption of the discontinuity of the shock front considered as the frontier between two states at thermodynamic equilibrium.

\subsection{Model of the Ideal Detonation}

The model considers (Figure 1a) a reactive supersonic flow, self sustained by the shock wave, steady as far as the end of the reactions : all the exo-energetic reactions are assumed to reach an equilibrium at the same time, this equilibrium being a function of the temperature and of the pressure. Beyond this point, an unstationnary rarefaction takes place being controlled by the boundary conditions of the explosive charge. In this downstream flow, the composition of the products may change as a function of $p, V, T$, but no energy can be transfered upstream and influence the characteristics of the detonation.

The set of the conservation equations to be solved in building the model neglect the conductive and radiation effects and assume a laminar non viscous flow.

The steady state condition means that the shock front and the plane of the end of reaction propagate at the same velocity $\mathrm{D}$ in a plane semi-infinite geometry. The indexes 0 and 1 correspond respectively to the non reacted and totally reacted material.

If we consider that the plane of the end of reaction is the head of an unstationnary rarefaction, the condition of a steady state upstream is fulfilled if this plane is sonic, $D=\hat{\mathfrak{u}}+\hat{a}$, where $\hat{\mathfrak{u}}$ and $\hat{a}$ are the material velocity and the sound speed at this plane. This condition was found by Chapman [1] and Jouguet [2] who have shown its consequences in the thermodynamic (p, v) plane (Figure $1 \mathrm{~b})$. In the (p, v) plane, 0 represents the fresh explosive and the locus of the possible final states of the reaction products is the curve $(\mathrm{H})$ obtained by solving the conservation equations :

$$
H(p, v)=1 / 2\left(p+p_{o}\right)\left(v_{o}-v\right)+E(p, v)-E_{O}\left(p_{o}, v_{O}\right)=0
$$




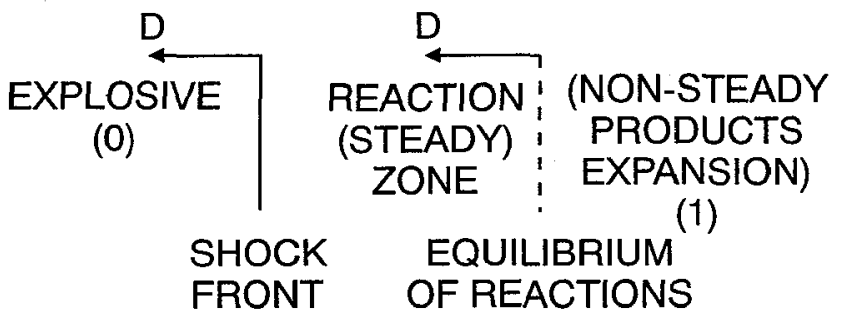

FIG. 1a: SPATIAL SCHEME

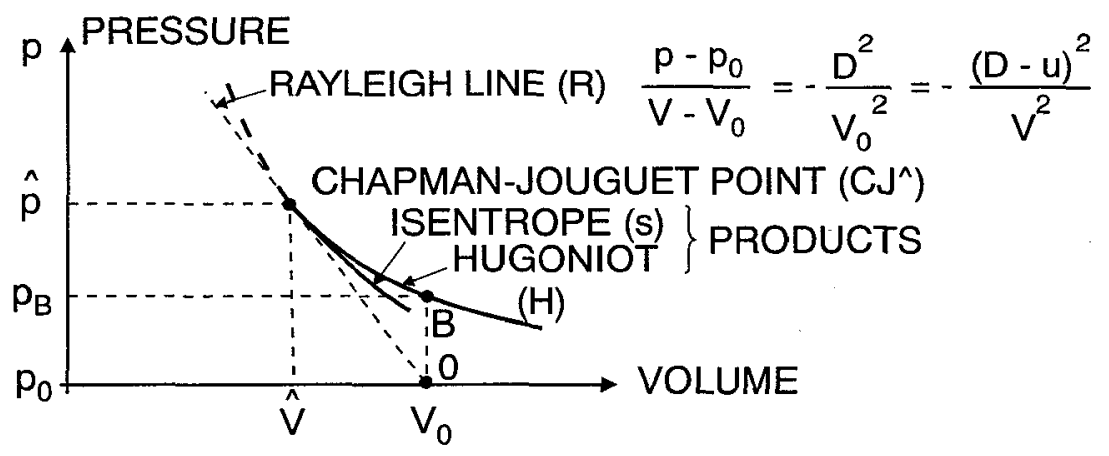

FIG. 1b : CHAPMAN-JOUGUET MODEL $(p, v)$

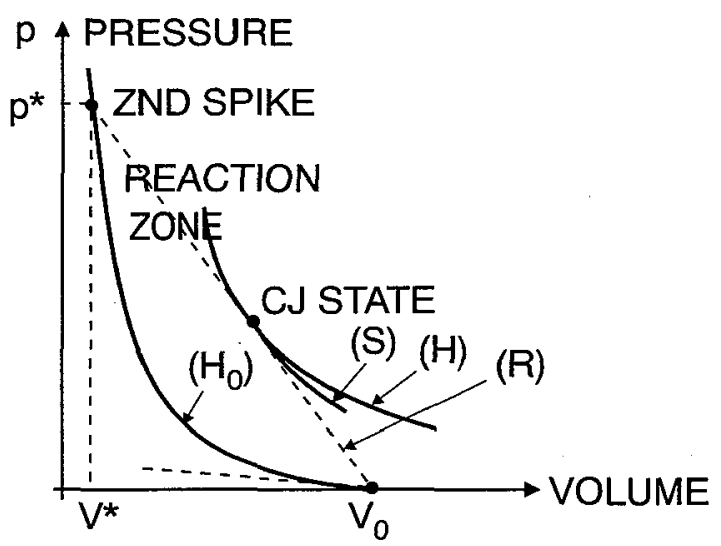

FIG. 1c : COMPLETE DESCRIPTION $(p, v)$

FIG. 1 : IDEAL THERMO-HYDRODYNAMIC DETONATION 
The basic difference from an Hugoniot for non-reacted explosive is in the meaning of $\mathrm{E}=$

$$
\begin{array}{ll}
\mathrm{E}_{\mathrm{O}} \quad=\mathrm{E}_{\mathrm{O}}^{\prime} & \begin{array}{l}
\text { (internal energy of the fresh material }+\mathrm{q}_{\mathrm{o}} \\
\text { (energy of formation of the explosive molecule) }
\end{array} \\
\mathrm{E} \quad=\mathrm{E}^{\prime} \quad \begin{array}{l}
\text { (internal energy of the reaction products }+\mathrm{q} \\
\text { (energy of formation of the products) }
\end{array}
\end{array}
$$

As a consequence $\left(\mathrm{p}_{\mathrm{O}}, \mathrm{v}_{\mathrm{O}}\right)$ is not on $(\mathrm{H})$ and the ordinate on $(\mathrm{H})$ for $\mathrm{V}=\mathrm{V}_{\mathrm{O}}$ is a point $\mathrm{B}$ such that $\mathrm{pB}_{\mathrm{B}}-\mathrm{p}_{\mathrm{o}}$ is the increase of the pressure for a constant volume reaction at $\mathrm{V}=\mathrm{V}_{\mathrm{O}}$.

By solving the conservation equations it is possible to express the velocities $D, u$ as functions of $p$ and $V$ :

$$
-\frac{D^{2}}{V_{o}^{2}}=-\frac{(D-u)^{2}}{V^{2}}=\frac{p-p_{0}}{V-V_{o}}
$$

As a consequence, any final state on $(H)$ corresponds a velocity $D$ as a result of the intersection of $(\mathrm{H})$ and of $(\mathrm{R})$ called the Rayleigh line.

It is shown that the steady state condition for the Ideal Detonation is fulfilled when (R) is tangent with $\mathrm{H}$; At the tangent point, called CJ point, (R) is also tangent with the isentrope $\mathrm{S}$ the slope of which is $-\frac{\mathrm{a}^{2}}{\mathrm{~V}^{2}}$. As a consequence, at the CJ point

$$
\mathrm{D}=\hat{\mathfrak{u}}+\hat{\mathrm{a}}
$$

It is also interesting to note that the $\mathrm{CJ}$ detonation velocity is the minimal velocity which can be reached.

Until now, the reaction zone has been neglected or assumed to have no thickness. It will be seen later (see part 2) that the present description of the Ideal Detonation is sufficient for computation of the detonation parameters.

\subsection{A more complete description : The ZND model}

A description of the complete structure of detonation with propagation of the shock in the fresh explosive has been proposed quasi-simultaneously in 1942 by Doering, Zeldovitch and von Neumann [3], [4], [5]. The construction in the (p, v) diagram needs to know the Hugoniot $\mathrm{H}_{\mathrm{O}}$ of the non reacted explosive (Figure 1c). 
The steady state condition and the use of the Rayleigh line $(\mathrm{R})$ show that $\left(\mathrm{p}_{\mathrm{O}}, \mathrm{v}_{\mathrm{O}}\right),\left(\mathrm{p}^{*}, \mathrm{v}^{*}\right)$ and $(\mathrm{p}, \hat{\mathrm{u}})$ are aligned on a straight line of slope equal to $-\frac{\mathrm{D}^{2}}{\mathrm{~V}_{\mathrm{o}}{ }^{2}}$. In the reaction zone the pressure drops steeply from a maximum value $\mathrm{p}^{*}$, called the ZND spike, to the CJ pressure $\hat{\mathrm{p}}$. The simplest interpretation of the model would be to calibrate the $\mathrm{p}^{*}-\hat{\mathrm{p}}$ segment in the extent of reaction $I$, from $I=0$ at $p^{*}$ to $I=1$ at $\hat{p}$. However, we enter here into the most controversial part of the model as we will see now.

\subsection{First consideration on the limits of the model}

The following considerations deal with the simplicity of the physical assumptions and with the reality of one dimensional plane and semi-infinite geometry.

a) The existence of a non-reacted Hugoniot is questionnable if out of equilibrium effects and early decomposition occur. The main consequence would be to modify the reaction scheme and would have a major influence in the build-up to detonation following a shock of a short duration.

b) The validity of the assumption of a single reaction rate and of the absence of diffusion effects between the species has been roughly considered in the comparison between computations and experiments and is used to divide the explosives in non-ideal and ideal compositions. This point will be examined in part 3 at the same time as the coincidence of a sonic state with the end of the exo-energetic reactions.

c) The validity of the plane semi-infinite assumption for most of charges designed for military and civil applications is a question of time and space scale : The thinner the reaction zone the better the assumption. It is mainly the requirement for insensitive explosives in the last decades which has made it necessary to re-examine the question and to bridge between non-ideal geometries and non-ideal explosives mainly where the model is used as a tool for the design of charges.

\section{References part 1}

[1] Chapman D.L., Lond.Edi.nb.Dubl.Phil.Mag. 47(1899) 90

[2] Jouguet E., J.Math.pures.Appl. Paris 60(1905) 347

[3] Zeldovich Ya.B., NACA Tech.Memo.(1950) Nº 1261 (also "Selected works" p. 411)

[4] von Neumann J., OSRD Report (1942) $\mathrm{N}^{\circ} 549$

[5] Döring W., Burkhardt G., Air Material Command Technical Report - F-TS-1227-IA (GDAM A9-T46)(1949) 


\section{APPLICATIONS OF CLASSICAL DETONATION THEORY} (W. C. Davis)

In the preceeding chapter Dr. Fauquignon has presented the classical theory of detonation. This simple theory is the basis for almost all calculations made for the design of devices driven by high explosives. Here we will examine how it is used, and describe some tests of the theory.

In some applications of explosives, say an aerial bomb, the important quantities for design are the velocity of the case fragments and the strength of the shock wave at relatively large distances for the bomb. The partition of energy between fragments and gas does not depend strongly on the details of the detonation and the interaction of waves with the metal, and is not at all sensitive to the method used to calculate the performance of the system. Many approximate methods [1] have been developed for such problems. Results of such calculations do not provide a test of the applicability of the theory. On the other hand, understanding small details of the system, such as spalling of the case of the bomb, require detailed calculations, and it is important to get the details of the flow of the detonation products reasonably correct. These calculations do provide a test of the applicability of the theory.

The classical model [2] gives the state of the detonation products at the end of the chemical reaction zone, and shows further that the state does not depend on the details of the chemical reaction, but simply on the energy released. The state, usually called the Chapman-Jouguet or CJ state and designated here by a subscript $j$, is described by

$$
\mathbf{p}_{j}=\frac{\rho_{0} \mathbf{D}^{2}}{\gamma_{j}+1} \quad \rho_{i}=\rho_{0} \frac{\gamma_{j}+1}{\gamma_{j}} \quad \mathbf{u}_{j}=\frac{\mathbf{D}}{\gamma_{j}+1} \quad \mathbf{c}_{j}=\frac{\gamma_{j} \mathbf{D}}{\gamma_{j}+1}
$$

where $p$ is the pressure, $\rho$ is the density and subscript 0 denotes the initial state, D is the CJ detonation wave velocity, $\gamma$ is the dimensionless sound velocity to be discussed below, $u$ is the particle velocity, and $\mathrm{c}$ is the sound velocity. The dimensionless sound velocity $\gamma$ is a thermodynamic state variable and is given by

$$
\gamma=\frac{\rho \mathbf{c}^{2}}{\mathbf{p}}
$$

with the square of the sound speed defined as $\mathbf{c}^{2}=(\partial p / \partial \rho)$, for all $\rho$ and $p$, not just at the $\mathrm{CJ}$ state. Note that $\gamma$ in hydrodynamics is not the ratio of specific heats, and is not a constant except in special cases.

The classical model has the detonation wave running at constant speed; therefore if one knows, or assumes, its location at an instant of time, one can calculate [3] its location and shape at a later time. The model gives the state of the detonation products at the wave front from the formulas above. The flow of the products, an inert flow with the only possible chemical changes being shifts in equilibrium composition as the state changes, can be computed from the usual equations [2] of hydrodynamics. The only processes that produce appreciable entropy are shock waves in the flow of the products; if there are no shocks, the flow is isentropic. If there are shocks, they move the state off the isentrope, in most cases only 
slightly. The material description needed to complete the formulation of the flow of the products gases is required only near the isentrope through the $\mathrm{CJ}$ point (called the principal isentrope).

While it may seem straightforward to model detonation by advancing the wave at each time step, and setting the state to the $\mathrm{CJ}$ state at that position, this procedure often causes numerical difficulties [4]. Since the state at the end of the chemical reaction is independent of the details of the reaction, any method for reacting the explosive to its products will give the same end state. In modeling, any scheme that reacts the explosive quickly and does not introduce numerical difficulties will be satisfactory. Many schemes have been used; some work better than others. Most calculations are made using one of the schemes for burning the explosive; there is no attempt to model the real reaction zone.

Any thoughtful person must ask why it is necessary to introduce this artificiality. Why not just model the reaction zone following all the details of the chemical reactions? The answer is that it is not practical to do that. To get even a reasonably accurate model of the reaction zone requires at least 30 calculational cells in the reaction zone. The device will usually have a characteristic size that is 100 to 1000 or more reaction zone lengths, to be far enough from the critical size, or failure size, for the system to be robust. While one-dimensional calculations are possible with 10000 or more cells, two- and three-dimensional calculations with $10^{8}$ or $10^{12}$ cells, the ones of interest, are not. Attempts to model the reaction zone in a real system take more than a day on the fastest computers.

Whether the front is advanced and the state set using the classical theory, or by using an artificial burning, a description of the properties of the detonation products is required in every calculation. The explosive driven system must be considered as an engine, differing from more conventional engines in that the parts are deformed as well as moved and that the cycle is not repeated. The detonation products are the working fluid for the engine. The description [5-7] of the products is usually called an "equation of state". The quantities that are required are two derivatives:

$$
\gamma(p, \rho) \equiv(\rho / p)(\partial p / \partial \rho)_{s} \quad \Gamma(p, \rho) \equiv(1 / \rho)(\partial p / \partial E)_{\rho}
$$

where $E$ is the specific internal energy. Values are needed only in the immediate neighborhood of the principal isentrope that passes through the CJ point, because little entropy is produced in the weak shocks that may occur in the system after detonation.

The material description for calculations is almost always obtained by calibrating a convenient fitting form to experiments as much like the system to be calculated as possible. Sometimes the fitting includes results from equations of state calculated by statistical mechanics using assumed intermolecular force laws, and other theoretical equations of state. Simple expansions of the equation of state such as a virial expansion are of limited use. The densities are so high that the intermolecular interaction energy is very large compared with the thermal energy. Typically in the region of most of the energy transfer from products to inert parts one has $\mathrm{p} / \mathrm{\rho RT}>10$. The results are presented as

$$
p=p(\rho, E)
$$


and the necessary derivatives for the hydrodynamic calculations are obtained from this expression. The "equation of state" is incomplete (a function of $\rho$ and $E$ rather than of $\rho$ and $S$ ) because it is calibrated from mechanical measurements that yield no information about temperature or entropy.

One sees from this description of how the classical theory is used, to provide a model for fitting the measurements, that its success for this purpose does not provide a real test of the theory. The fact that the calibration experiments can be quite different from the system being modeled indicates that the classical model is probably qualitatively correct. A definitive test must use other data.

The first reassuring fact $[8,9]$ is that most, but not all $[10,11]$, detonations do in fact propagate with a leading shock wave that is reasonably smooth, followed by a decrease in pressure as the chemical reaction takes place, and then, if the back boundary condition is proper, followed by an expansion wave. These observations show that the classical model is at least qualitatively correct. The measured reaction zone in practical explosives ranges from about $10 \mu \mathrm{m}$ to about $10 \mathrm{~mm}$, a range of 1000 . Explosives with reaction zones smaller than $10 \mu \mathrm{m}$ are too dangerous to handle safely, and ones with reaction zones larger than $10 \mathrm{~mm}$ fail to propagate except in very large sizes.

Experiments [12] providing a severe test of the theory were carried out using liquid nitric oxide NO as a prototype explosive. The description of the detonation products was obtained from shock wave measurements made on liquid oxygen, liquid nitrogen, and liquid mixtures of oxygen and nitrogen. A careful study, using the best theory available to help the analysis was made. No calibration to detonation measurements was required. Then the results of measurements of detonation velocity and $\mathrm{CJ}$ pressure were compared with the predictions of the theory, and the agreement was found to be very satisfactory. The really important conclusion to be reached, which could not be supported otherwise, was that the assumption that the detonation products reach chemical and thermal equilibrium in the detonation reaction zone is an excellent approximation.

Another set of experiments [13], however, testing the theory by the "inverse method", showed that the theory is not exactly correct. This method requires that the initial state of the explosive is varied to provide values for derivatives of detonation velocity with respect to initial density and energy about a standard initial state. For TNT the energy was varied by using solid and liquid TNT so the energy was different by the heat of fusion, and the density was varied by using solid TNT pressed to different densities, or by varying the temperature of the liquid. For liquid nitromethane the energy was varied by mixing it with a mixture of nitric acid, acetonitrile, and water that had the same atomic composition but different energy, and the density was varied by changing the temperature. The results for the derivatives allowed the pressure to be calculated, and this inferred value was compared with measurements of detonation pressure. In these experiments agreement was not obtained; the values differed by several standard deviations.

The main support for belief that the classical theory is a good approximation to actuality comes from its use in predicting the behavior of an explosive, perhaps even an explosive that has never been made. It was recognized [14] at least 75 years ago that if the simple theory is correct one need know only the atomic composition of the explosive and its heat of formation 
to calculate its detonation properties. The atomic composition determines the composition of the products, since they are (by assumption) in chemical equilibrium. Most of the heat comes from the formation of the products, so the dependence on an exact figure for the heat of formation of the explosive is relatively weak. For most common explosives, the major products are $\mathrm{N}_{2}, \mathrm{H}_{2} \mathrm{O}, \mathrm{CO}_{2}, \mathrm{CO}$, and $\mathrm{C}$ (soot), with minor amounts of $\mathrm{NO}, \mathrm{NH}_{3}, \mathrm{CH}_{4}, \mathrm{H}_{2}, \mathrm{O}_{2}$, $\mathrm{HCOOH}$, etc. Sometimes other elements besides $\mathrm{CHNO}$ are present. If an equation of state for each species is available, and a mixing rule is known, the free energy can be minimized to give the composition of the products and the state variables. There need be no calibration to detonation experiments.

While simple in concept, this procedure is complex and tedious in execution. Many workers [15-17] have made schemes for doing it, and many equations of state with various calibration data have been used, and different mixing rules have been tried. Other minor constituents have been considered. Obviously, different schemes give slightly different results. However, all reasonable schemes give surprisingly good predictions, usually within a few per cent of the measured values. Rarely in science and engineering has it been possible to make predictions of the detailed properties of complex materials as well as has been done for explosives. The value of the classical simple theory is proven by these results.

Reading the current papers on explosives one soon sees that workers are not satisfied with the available predictions. To make a decision about whether or not to invest the large amount of effort and funds needed to develop a new explosive for practical application, the accuracy of a few per cent is not good enough. The gain in performance from a new explosive is never more than a few per cent above the old ones. While refining the equations of state and the mixing rules, or trying new minor constituents, seems to help a little, studying the deficiencies and errors soon leads one to believe that there is an underlying additional cause of error. The most likely cause is that the products do not go completely to equilibrium in a short enough time in all explosives. There is strong evidence for very slow heat release near the end of the reaction zone. Perhaps the slow reactions can be identified and predicted, with subsequent improvement of the prediction of explosive performance.

This brief review describes how the classical theory of detonation, in its simplest form, is applied to practical problems. Almost all calculations of the behavior of explosive driven systems are based on the theory. Predictions of the performance of new explosives are based on the theory. The theory has been tested in many ways, not all of them described here. It is not perfect, nor is it complete, but it is extremely useful and is very widely used.

\section{References part 2}

[1] G. E. Jones, J. E. Kennedy, and L. D. Bertholf, Ballistic calculations of R. W. Gurney, Am. J. Phys. 48, 264-9 (1980).

[2] W. Fickett and W. C. Davis, Detonation, U. of Califomia Press, 1979, Chap. 2.

[3] J. B. Bdzil and D. S. Stewart, Modeling two-dimensional detonations with detonation shock dynamics, Phys. Fluids A 1, 1261-7 (1989), Sect. IIIA. For more detail, see J. Bdzil and W. Fickett, DSD technology : a detonation reactive Huygens code, Los Alamos Report LA-12235-MS, 1992.

[4] C. L. Mader, Numerical modeling of detonation, U. of California Press, 1979.

[5] W. C. Davis, Equation of State for Detonation Products, Eighth Symposium (International) on Detonation, NSWC MP 86-194, 1985, pp. 785-795. 
[6] R. Menikoff and B. J. Plohr, The Riemann problem for fluid flow of real materials, Rev. Mod. Phys. 61, 75-130 (1989).

[7] W. C. Davis, Equation of state for detonation products, Tenth Symposium (International) on Detonation, preprints pp. 112-3, 1993.

[8] J. B. Bdzil and W. C. Davis, Los Alamos Report, LA-5926-MS, 1975.

[9] J. B. Bdzil, Perturbation Methods applied to problems in detonation physics, Sixth Symposium (International) on Detonation, ACR-221, 1976, pp. 352-370.

[10] L. V. Altshuler, Shock waves and extreme states of matter, Shock Compression of Condensed Matter, Williamsburg APS Meeting, 1991, pp. 3-14. See Figs. 2 \& 3.

[11] W. Fickett and W. C. Davis, Detonation, U. of California Press, 1979, Chap. 5.

[12] G. L. Schott, W. C. Davis, and W. C. Chiles, Initiation and detonation measurements on liquid nitric oxide, Ninth Symposium (International) on Detonation, OCNR 113291-7, 1989, pp. 1335-1345.

[13] W. C. Davis, B. G. Craig, and J. B. Ramsay, Failure of the Chapman-Jouguet theory for liquid and solid explosives, Phys. Fluids 8, 2169-2182 (1965).

[14] R. Becker, Impact waves and detonations, Zeit. Phys. 8, 321-362 (1922), English trans. NACA TM 505 and TM 506, March 1929.

[15] J. Taylor, Detonation in condensed explosives, Clarendon Press, 1952.

[16] C. L. Mader, Numerical modeling of detonations, U. California Press, 1979, Chap. 2.

[17] Detonation Symposium Volumes. There are more than 150 papers listed under the heading EOS in the index to the first nine Symposia.

\section{DETONATION IN A NON IDEAL GEOMETRY}

(C. Fauquignon)

Some usual cases are :

- Divergent and convergent 1-D detonation (the planar condition is not respected)

- Steady detonation in a cylindrical stick

- Mach detonation : In theory it is a special 1D detonation, but its existence results from $2 \mathrm{D}$ boundary conditions.

We shall concentrate here on the steady detonation in a cylindrical stick for which a large amount of accurate experimental results on the detonation velocity and on the front curvature as a function of the diameter is available.

\subsection{Sketch of the reactive flow}

Figure 2a features some stream lines, the curved shock front, the sonic surface which corresponds to a barrier for acoustic waves propagating upstream toward the shock front, the surface where the reactions end. 


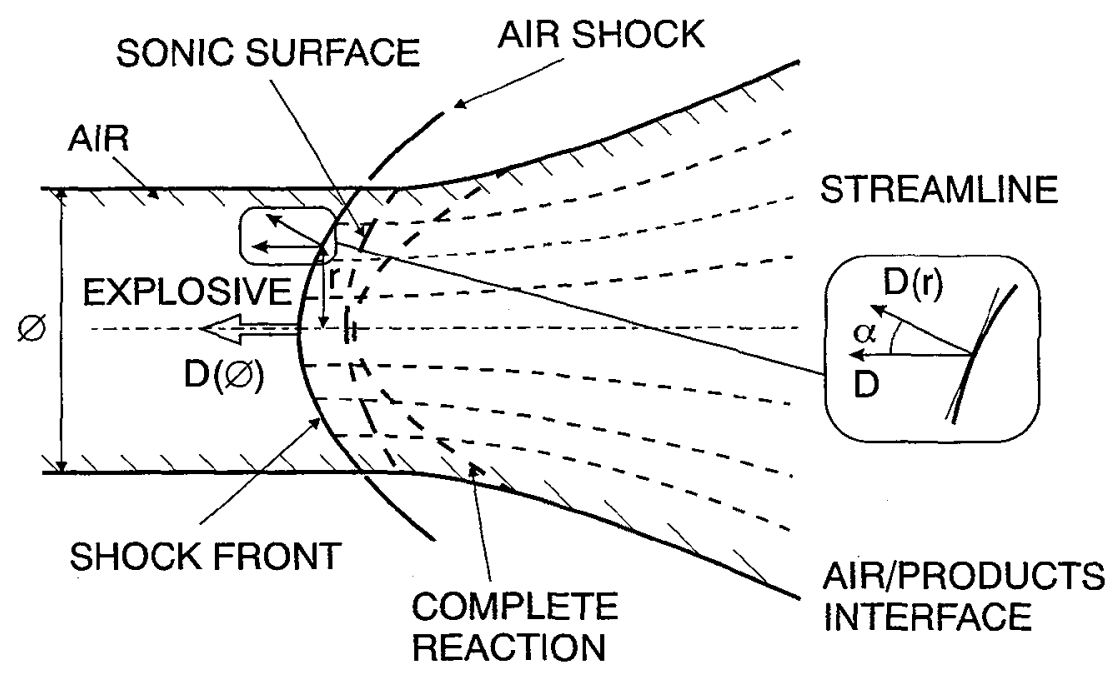

FIG. 2a : PHENOMENOLOGY OF THE REACTIVE FLOW

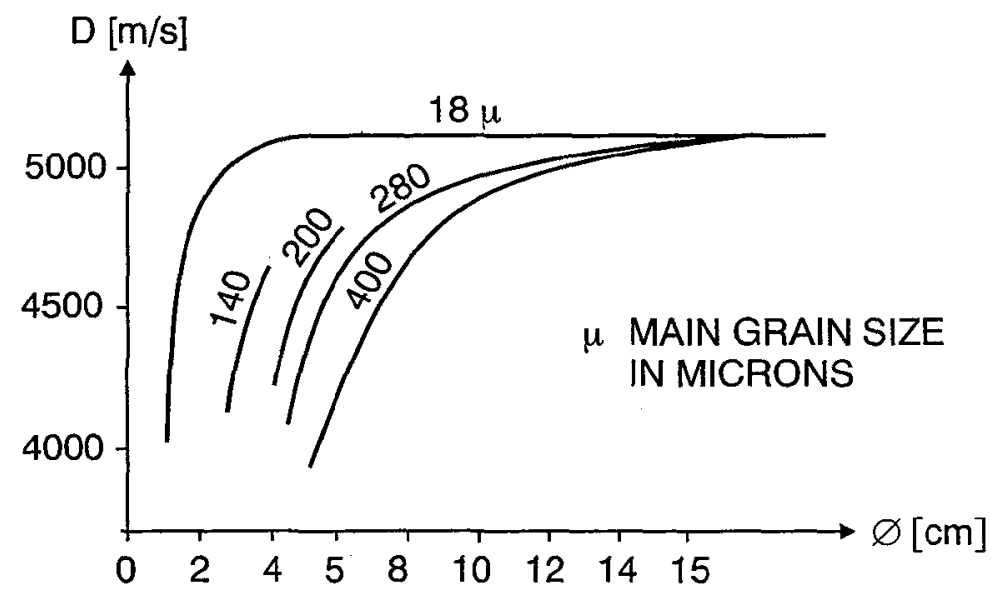

FIG. $2 b$ : DETONATION VELOCITY D VERSUS STICK DIAMETER $\varnothing$ PRESSED TNT $\rho_{0}=1.2 \mathrm{~g} \times \mathrm{cm}^{-3}$

FIG. 2 : DETONATION OF A CYLINDRICAL STICK 
The local curvature of the front determines the initiation conditions for the reactions along the stream line passing through this point. The intensity of the shock is given by its velocity $D \cos \alpha$. In the steady self-sustained detonation, the shock intensity also depends on the energy delivered on the stream line between the shock and the sonic surface. The energy delivered downstream between the sonic surface and the surface of end of reactions mainly supplies the lateral expansion.

As the diameter of the stick decreases, the part of the energy delivered to sustain the shock decreases and, finally the failure diameter is reached.

A first observation is that the steady state is not strictly governed by the sonic condition but depends strongly on the lateral expansion. A second observation is that the detonation prediction, for instance of its velocity, depends not only on the reaction kinetics, on the equation of state of the intermediate species (needed to calculate the sound velocity) but also on the thermo-mechanical processes involved in the shock front to initiate the reactions.

As a consequence of the overlapping of all these factors, most of them being poorly known, the theoretical and numerical approaches call upon global phenomenological models with fitting parameters based on detonation velocity or front curvature measurements.

In the case of the physically heterogeneous solid explosives, approaches similar to those used in Shock to Detonation Transition analysis (see 3.4) are performed. They use the three step scheme :

(a) Formation of hot spots

(b) Collapse of the reacting hot spots

(c) Burning.

Sometimes this scheme is concentrated in a two step process, Ignition and Build up.

\subsection{Some experimental observations}

One of the first observations is the major role of the physical heterogeneities illustrated by Figure $2 b$ which represents the velocity versus the stick diameter for different TNT grain sizes, with the loading density, i.e. the energy, being constant. To interpret the results it is to be noted that the grain size has opposite effects in the three step scheme. The larger the grain size the more energetic are the hot spots but the longer is the burning time. Such experimental observations have been used some decades ago, to test a grain burning model with arbitrary burning rate function of the pressure, but as the role of the grain size in the ignition was neglected the agreement with the experiment was relatively poor.

An interesting experimental study aiming at an independant variation of the influence parameters of (a) and (c) has been performed using a mixture of nitromethane either with a physical sensitizer (silica particles) or with a liquid chemical catalyst the diethylene-triamine (DETA) [1] which influences only the burning rate. 


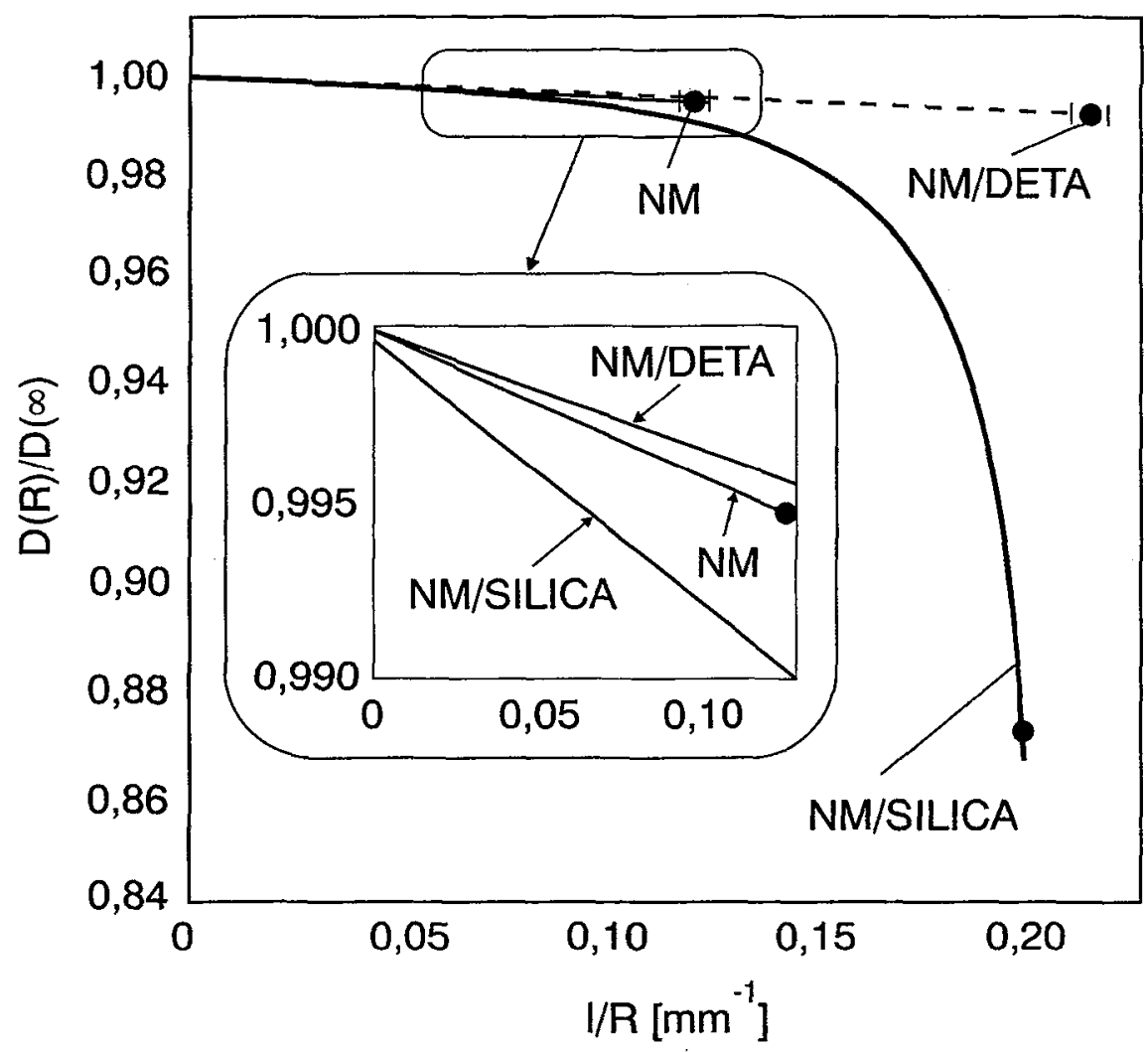

FIG. 3 : DETONATION VELOCITY OF NITROMETHANE (NM) MIXTURES VS RADIUS OF THE CHARGE 
Figure 3 presents the results which confirm the expected phenomenology :

- The silica particles absorb a part of the explosive energy but allow the detonation to be self sustained even at low detonation velocities, i.e. at low shock pressure.

- DETA shortens the burning time, i.e. the reaction zone, without changing the energy much.

- Both agents allow the mixtures to reduce the failure diameter to comparable values as they follow independent modes of action.

In spite of the large amount, the variety and the quality of the experimental observations, they give global information, the detonation velocity and the front curvature, and can only be used in the frame of an a priori scheme.

The present studies concerning the hot spots which will be presented in this meeting will help to a better understanding of the initial conditions in the shock front of most of the military and civil compositions. However, the transfer of the energy to the bonds of the molecules remains a question which cannot be tackled with the same methods due to a major difference in the time scale of the thermo-mechanical and of the physico-chemical processes.

\subsection{Theoretical approaches}

Specific studies aim mainly at taking into account multi-dimensionnal effects, using as a reference the plane $\mathrm{CJ}$ or $\mathrm{ZND}$ model, in order to improve the codes for numerical modeling.

These models refer to the fluid mechanics applied to an homogeneous material, and to a simple single reaction rate. They mainly differ in the mathematical formulation of multidimensionnal effects represented by transverse waves and flow along the curved front surface.

A description of these studies is beyond of the aim of this meeting and we just mention some recent references [2], [3]. However, following a similar path, L. Brun has brought a new generalized mechanical description of the detonation which will be presented in this meeting.

As has been noted before the reactive flow is too complex to evidence any chemical kinetics law directly from the experiment. An a priori prediction of a detonation in a multidimensionnal geometry requires to use in the numerical code a kinetics law determined in a specific experiment and it seems that the Shock to Detonation Transition (SDT) in plane geometry is the best way to get it.

\subsection{The shock to detonation : A time dependant process}

The SDT in an efficient tool to analyse the detonation mechanism for at least three reasons :

- The initial conditions, i.e., the shock pressure and duration are well defined and can vary from one experiment to another. 

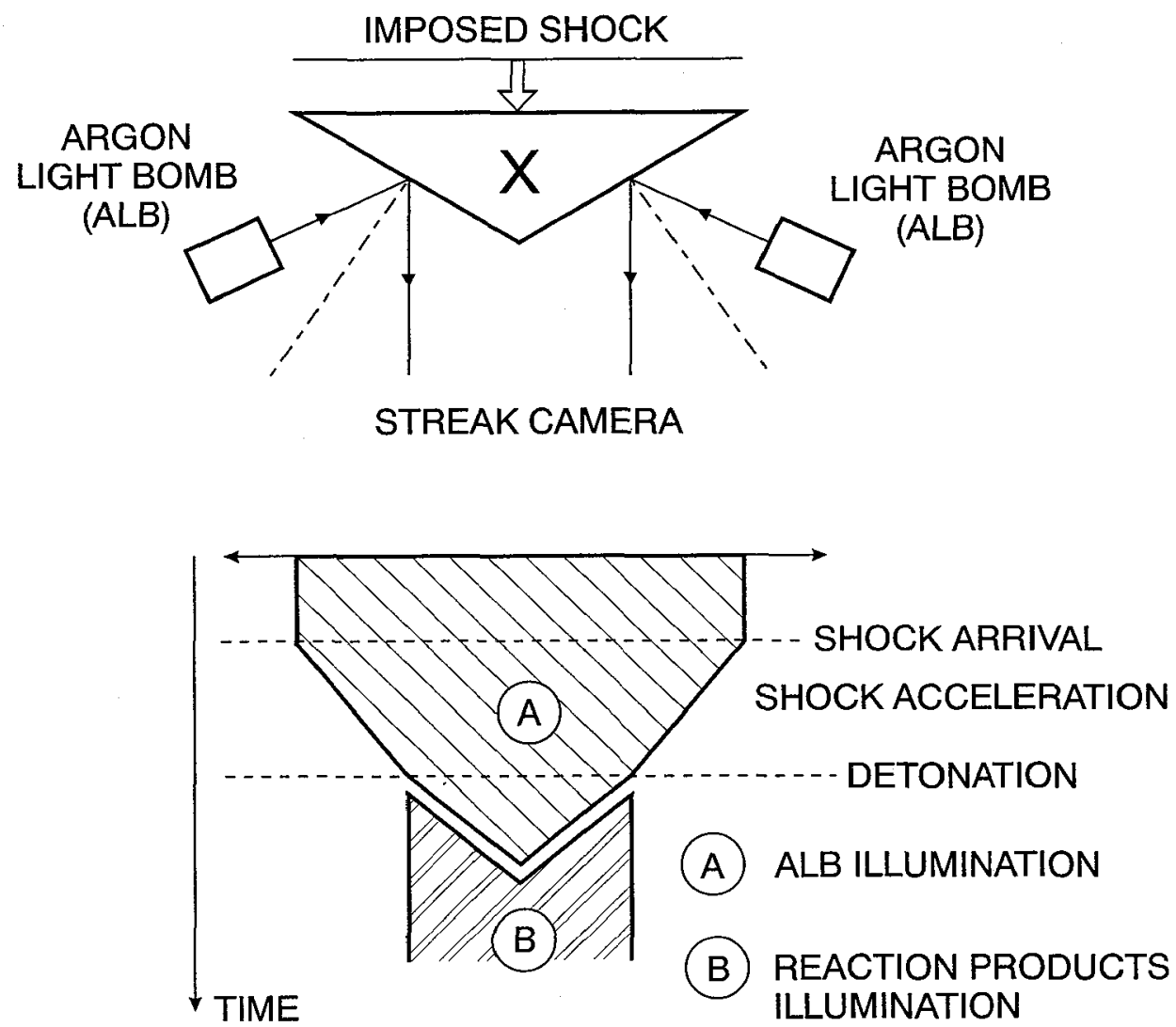

FIG. 4 : EXPERIMENTAL STUDY OF SDT BY THE DOUBLE EXPLOSIVE WEDGE TECHNIQUE 
- At pressures much lower than the ZND pressure, the SDT experiment gives an enlarged picture of the reactive flow from the initial shock compression up to a quasi steady detonation.

- The experiment requires a small amount of explosive that is suitable for fine measurements.

For about 35 years, the wedge technique (Figure 4) [4] has been used with some refinements.

The phenomenological reactive models which have been developed using measurements of the path vs time of the accelerating reactive shock [5] are based on the two step ignition and build up process for explosives assuming that hot spots are responsible of the initial heating. A survey of some of this models is given in [6].

Lagrangian analysis of the pressure or of the particle velocity in the build up zone is also used with success to check the validity of reactive models.

In spite of the efficiency of the method a more accurate knowledge of the initial conditions behind the shock front, i.e. of the microscopic heating processes and of the microstructure of the explosive specimens or of some out of equilibrium molecular processes is required to get a true chemical kinetics law. However, the reactive models are efficiently used by the designers of charges after having fitted the parameters with SDT experiments on the explosive composition to be used.

\section{References part 3}

[1] Engelke R., Phys. Fluids 22 (1979) pp. 623-630

[2] Bdzil J.B., Fickett W., 9th Symposium on Detonation - OCNR 113291-7 (1989) pp. $730-742$

[3] Lambourn B.D., Swift D.C., 9th Symposium on Detonation - OCNR 113291-7 (1989) pp. 784-797

[4] Campbell A.W., Davis W.C., Ramsay J.B., Travis J.R., Phys. Fluids 4(1961) pp. 511521

[5] Moulard H., Kury J.W., Delcos A., 8th Symposium on Detonation (1985) NSWC-MP 86-194 Report, pp. 902-913

[6] Fauquignon C., Journal de Physique, Colloque C4 Supplément au $\mathrm{n}^{\circ}$ 9, Tome 48, Sept. 1987 , pp. 39-65.

\section{4 - FRONTIERS OF DETONATION THEORY}

(W.C. Davis)

All detonation studies involve the interaction of chemistry and mechanics. In the detonation reaction zone the flow is not smooth and laminar, and consequently the shock wave is not smooth. The result is that the explosive is not heated uniformly, and the chemical reaction rate, an extremely non-linear function of temperature, is much more non-uniform. Reaction is fast in some small regions, usually called hot spots, and, relatively, almost nonexistent elsewhere until it spreads from the hot spots. 
In cast solid explosives, pressed polycrystalline explosives, emulsions and slurries, and in loosely packed powders, most of the hot spots are in the neighborhood of lower density regions. Where the density is low, the motion is greater, and more work is done on the material by the motion. The internal energy rises there, and the temperature is higher. The scale of the hot spots is related to the size of the crystallites or the rate of cooling when casting, etc., and these sizes are determined by such considerations as convenience for handling. How much reaction takes place at a hot spot depends of the rate of reaction and the rate of diffusion of heat away from the hot spot. In two explosives with crystallites the same size but reaction rates very different, the apparent effect of the hot spots may be very different.

In liquid explosives, it might seem that there will be no hot spots. Nature is not so kind. The flow in a detonation in a liquid explosive is still not smooth and laminar, because the burning is hydrodynamically unstable [1], and generates transverse waves spontaneously. (The solid explosives discussed in the preceeding paragraph also have these instabilites, and they compete with the ones generated by inhomogeneity). The scale and the spacing of these hot spots is related to the scale of the chemical reaction zone; the details are not understood, and are a subject for future research. The structures that have been studied seem to have a spacing of a few, 3 to 10 , reaction zone lengths.

Detonation in gases [1] produces very strong transverse waves, often with a shock wave collision called a Mach stem, where the pressure and temperature are far above the average. In some cases almost half the gas flows through this interaction region. It is as if the detonation, unable to propagate in a laminar flow, created superchargers for itself to ensure propagation. Gas detonations have been studied extensively (they do not destroy the equipment, the equations of state are known, the flow is transparent, and the transverse wave structures can be made large by reducing the initial pressure) and much is known about the details of the transverse waves. It is tempting to believe that structures in condensed phase explosives are analogous to those in gases. After all, if one explosive is not more like another explosive than anything else, there can be no science of explosives. But probably gases are different from liquids and solids. The spacings are 100 or more reaction zone lengths. The mechanical details are very different because of the differences in the equations of state.

The published papers [2] on detonation abound with papers on hot spots. It has been known for a very long time that imperfections and inhomogeneities strongly influence the critical size below which detonation will not propagate, and that many explosives must have hot spots to be useful. Two batches of explosive with identical chemical composition may differ enough in their hot spot behavior to make one batch useful and the other useless. Obviously, producers of explosives have learned to control the hot spots. The knowledge is all empirical.

One result of the current inability to model the effects of all the varied phenomena lumped together under the heading hot spots is that there is no way to take any available knowledge of chemical reaction rates for well-defined chemical explosive and predict what a particular formulation will do. For example, in one long list comparing the sensitivity of explosives in the gap test, a cast TNT formulation was the least sensitive explosive, and a pressed TNT formulation was the most sensitive. When the behavior of the explosive is accurately described by the simple classical theory discussed in Parts $1 \& 2$ of this introduction, that is, 
when the effective reaction zone length is small relative to system dimensions, the reaction rate has little effect on performance. Part 3 made it clear that safer explosives with long reaction zones cannot be described so simply, and the details of the phenomena in the reaction zone are important. When transient behavior is important, as in initiation of detonation intentionally, and even more in accidental initiation, the reaction zone in all its multidimensional detail, is even more important.

The difference between the classical theory of Parts $1 \& 2$, and the more complex ideas of Part $3 \& 4$, is that the classical model has only one important space scale, the reaction zone length. In most of Part 3 the dimensions of the charge provide another space scale. Above in Part 4 a scale of inhomogeneity was added. There are many space scales that are important in some detonation problems; fortunately not all of them are important in all detonation problems. When the flow is steady or nearly steady, that is, a wave moves but changes only slowly with time, space and time scales are simply related. When the transients are considered, space and time scales are not so neatly interchangeable.

A list of some important space scales is given in Table I. It is just an evaluation of the order of magnitude of these scales, and is arbitrary in many respects. However, it shows an enormous range. Modeling, whether analytical or computational, for systems where the ratio of important space scales is large presents great difficulties.

New developments in detonation theory are needed to incorporate treatments of all the important processes at their disparate scales. The new approaches discussed in Part 3 are a start. This meeting, with its title "Microscopic and Macroscopic Approaches to Detonation", is directed to the frontiers of detonation science.

TABLE I

\begin{tabular}{|l|l|}
\hline Item & Scale(meter) \\
\hline Charge size & \\
\hline Reaction zone length & $10^{-1}$ \\
\hline Crystallites, dendrites, emulsions & $10^{-5}-10^{-2}$ \\
\hline Hot spots & $10^{-5}-10^{-3}$ \\
\hline Shock roughness & $10^{-7}-10^{-4}$ \\
\hline Shock thickness & $10^{-7}-10^{-4}$ \\
\hline Atoms and molecules & $10^{-8}$ \\
\hline
\end{tabular}




\section{References Part 4}

[1] W. Fickett and W. C. Davis, Detonation, U. California Press, 1979, Chaps. 6 \& 7.

[2] S. L. Crane, W. E. Deal, J. B. Ramsay, A. M. Roach, and B. E. Takala, Index for the proceedings of the Symposia (International) on Detonation, 1951-1989, Tenth Symposium (International) on Detonation, preprints p. 473, 1993. There are 75 entries under the heading "hot spots". 